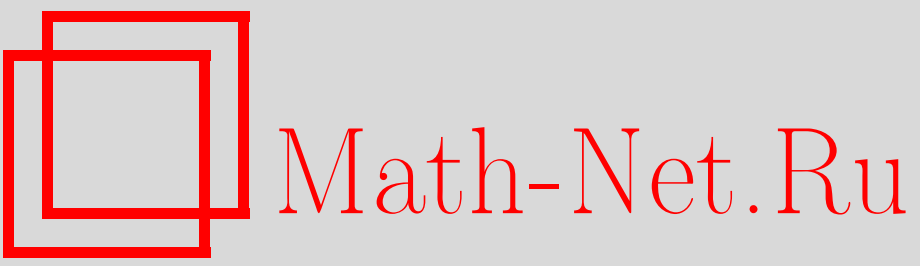

А. Г. Терентьев, Об одном классе линейных отображений и их положительной обратимости, УМН, 1996, том 51, выпуск 6, 223-224

DOI: https://doi.org/10.4213/rm1038

Использование Общероссийского математического портала Math-Net.Ru подразумевает, что вы прочитали и согласны с пользовательским соглашением

http://www.mathnet.ru/rus/agreement

Параметры загрузки:

IP: 3.80 .181 .102

26 апреля 2023 г., $17: 55: 48$ 


\title{
ОБ ОДНОМ КЛАССЕ ЛИНЕЙНЫХ ОТОБРАЖЕНИЙ И ИХ ПОЛОЖИТЕЛЬНОЙ ОБРАТИМОСТИ
}

\author{
A. Г. ТЕРентьЕВ
}

Пусть $X$ и $Y$ - отделимые локально выпуклые векторные топологические пространства над недискретным полем $K, E$ - подпространство $X$. Мы рассмотрим класс $\Omega$ линейных отображений $T_{\alpha}: E \subset X \rightarrow Y$ (с областью определения $D\left(T_{\alpha}\right)=E$, со значениями в $Y$ ), имеющих вид: $T_{\alpha}=B-A_{\alpha}$, в предположении, что в этом классе существует хотя бы одно биективное отображение $T_{\beta}$, являющееся неким эталоном.

Если $X$ и $Y-F$-пространства, а отмеченное эталонное отображение $T_{\beta}$ замкнуто, то обратное отображение $T_{\beta}^{-1}: Y \rightarrow X$ непрерывно [1, с. 116], и, таким образом, класс $\Omega$ содержит корректные по Адамару отображения (например, отображения, возникающие в краевых задачах для функционально-дифференциальных уравнений, [2], [3, гл. III].

1. Главными из свойств, которыми обладают отображения $T_{\alpha} \in \Omega$, являются следующие два свойства $\left(T_{\beta}\right.$ - эталонное отображение):

$$
\begin{array}{ll}
T_{\beta}^{-1} T_{\alpha}=I-L_{\alpha \beta}, & L_{\alpha \beta}=T_{\beta}^{-1}\left(A_{\alpha}-A_{\beta}\right), \\
T_{\alpha} T_{\beta}^{-1}=I-R_{\alpha \beta}, & R_{\alpha \beta}=\left(A_{\alpha}-A_{\beta}\right) T_{\beta}^{-1},
\end{array}
$$

где $I$ - тождественньй оператор, а $L_{\alpha \beta}: E \rightarrow E$ и $R_{\alpha \beta}: Y \rightarrow Y$ - линейные операторы.

ОПРЕДЕЛЕНИЕ. Отображение $T_{\alpha}$ мы называем левым (соотв. правым) почти фредгольмовым, если оно обладает свойством (Л) (соотв. (П)).

Если один из операторов $L_{\alpha \beta}$ или $R_{\alpha \beta}$ является, например, потенциально компактныц (т.е. некоторая его целая положительная степень компактна), то отображение $T_{\alpha}$ в точности оказьвается фредгольмовым (т.е. оно нётерово и его индекс равен нулю; мы следуем [4]).

Через $\widetilde{Z}$ обозначим векторное пространство всех линейных непрерывных операторов $A: Z \rightarrow Z$, наделенное некоторой локально выпуклой топологией. Ниже мы считаем, что $L_{\alpha \beta} \in \widetilde{E}$ и $R_{\alpha \beta} \in \widetilde{Y}$.

Если единица не является точкой спектра одного из операторов $L_{\alpha \beta} \in \widetilde{E}$ или $R_{\alpha \beta} \in \widetilde{Y}$, то она не является точкой спектра и для другого оператора. При этом справедливы следующие соотношения:

$$
\begin{aligned}
& \left(1^{\text {л,п }}\right) \quad\left(I-L_{\alpha \beta}\right)^{-1}=I-L_{\beta \alpha} ; \quad\left(I-R_{\alpha \beta}\right)^{-1}=I-R_{\beta \alpha} ; \\
& \left(2^{\pi, \Pi}\right) \quad L_{\alpha \beta}+L_{\beta \alpha}=L_{\alpha \beta} L_{\beta \alpha}=L_{\beta \alpha} L_{\alpha \beta} ; \quad R_{\alpha \beta}+R_{\beta \alpha}=R_{\alpha \beta} R_{\beta \alpha}=R_{\beta \alpha} R_{\alpha \beta} \text {; } \\
& \left(3^{\text {ग, }) ~} \quad L_{\alpha \beta}=T_{\beta}^{-1} R_{\alpha \beta} T_{\beta} ; \quad R_{\alpha \beta}=T_{\beta} L_{\alpha \beta} T_{\beta}^{-1}\right. \text {. }
\end{aligned}
$$

2. Дальнейшие рассуждения мы проводим для правых почти фредгольмовых отображений $T_{\alpha} \in \Omega$ (соотношения с буквой " $\Pi$ "). Ниже считаем $X$ и $Y$ вещественными $B$-пространствами. Мы предполагаем также, что пространство $Y$ наделено структурой порядка с помощью $c o 6 c m$ венного порождающего и нормального конуса $C_{Y}$ (здесь мы следуем [5]).

Структуру порядка в $E$ введем с помощью конуса $C_{Y}$ и эталонного отображения $T_{0} \in \Omega$, полагая $C_{E}=T_{0}^{-1} C_{Y}$. Ясно, что $C_{E}$ - конус в векторном пространстве $E$. Следовательно, $E$ наделяется структурой порядка с помошю конуса $C_{E}$ обычным образом. Поэтому дальше мы используем термины "положительное", "положительно обратимое", относя их к отображению $T$ в общепринятом смысле: $T C_{E} \subset C_{Y}, T^{-1} C_{Y} \subset C_{E}$. Для отношения порядка мы используем знак $\leqslant$.

Для отображения $T_{\alpha} \in \Omega$ отсюда и из свойства правой почти фредгольмовости (П) имеем:

ПредЛожение 1. Отображение $T_{\alpha} \in \Omega$ положительно обратимо тогда и только тогда, когда существует положительно обратимое отображение $T_{\beta} \in \Omega$ такое, что оператор $I-R_{\alpha \beta}$ положительно обратим. 
ПрЕДЛОЖЕнИЕ 2. Отображение $T_{\alpha} \in \Omega$ положстельно обратимо тогда и только тогда, когда существует положительно обратимое отображение $T_{\beta} \in \Omega$ такое, что оператор $R_{\alpha \beta} \in \widetilde{Y}$ положителен (т.е. $\left.R_{\alpha \beta} \in C_{\widetilde{Y}}\right)$ и $\rho\left(R_{\alpha \beta}\right)<1$. При этом $0 \leqslant T_{\beta}^{-1} \leqslant T_{\alpha}^{-1}$.

Действительно, в части $(\Rightarrow)$ следует положить $T_{\beta}=T_{\alpha}$, в части $(\Leftarrow)$ из $(\Pi)$ имеем: $T_{\alpha}^{-1}=T_{B}^{-1}\left(I-R_{\alpha \beta}\right)^{-1}$. Последнее неравенство следует из предложения 1 и соотношения

$$
T_{\alpha}^{-1}-T_{\beta}^{-1}=T_{\beta}^{-1}\left(I-R_{\alpha \beta}\right)^{-1} R_{\alpha \beta} .
$$

Следующее утверждение, называемое теоремой сравнения, решает вопрос о "грубости" свойства положительной обратимости линейных отображений класса $\Omega$.

Теорема 1. Пусть отображения $T_{\alpha}, T_{\beta}, T_{\gamma}$ класса $\Omega$ таковы, ито $T_{\alpha} \leqslant T_{\beta} \leqslant T_{\gamma}$ (или, что то же, $A_{\gamma} \leqslant A_{\beta} \leqslant A_{\alpha}$ ). Пусть, далее, отображение $T_{\gamma}$ положстельно обратимо и $\rho\left(R_{\alpha} \gamma\right)<1$. Тогда отображения $T_{\alpha}$ и $T_{\beta}$ положительно обратимь $и$ имеют место неравенства: $0 \leqslant T_{\gamma}^{-1} \leqslant T_{\beta}^{-1} \leqslant T_{\alpha}^{-1}$.

ДокАЗАтЕльСтво. а) Так как $T_{\alpha} T_{\gamma}^{-1}=I-R_{\alpha \gamma}, R_{\alpha \gamma}=\left(A_{\alpha}-A_{\gamma}\right) T_{\gamma}^{-1}$, то положительная обратимость отображения $T_{\alpha}$ и неравенства $0 \leqslant T_{\gamma}^{-1} \leqslant T_{\alpha}^{-1}$ следуют из условий теоремы и предложения 2.

б) Так как $T_{\beta} T_{\gamma}^{-1}=I-R_{\beta \gamma}, R_{\beta \gamma}=\left(A_{\beta}-A_{\gamma}\right) T_{\gamma}^{-1}$ и $R_{\alpha \gamma}-R_{\beta \gamma}=\left(A_{\alpha}-A_{\beta}\right) T_{\gamma}^{-1}$, то $0 \leqslant R_{\beta \gamma} \leqslant R_{\alpha \gamma}$. Отсюда и из нормальности конуса $C_{\widetilde{Y}}$ в $B$-алгебре $\widetilde{Y}$ следует, что $\rho\left(R_{\beta \gamma}\right)<1$. Теперь из предложения 2 следуют положительная обратимость отображения $T_{\beta}$ и неравенства $0 \leqslant T_{\gamma}^{-1} \leqslant T_{\beta}^{-1}$.

в) Так как $T_{\alpha} T_{\beta}^{-1}=I-R_{\alpha \beta}, R_{\alpha \beta}=\left(A_{\alpha}-A_{\beta}\right) T_{\beta}^{-1}$, то из обратимости отображения $T_{\alpha}$ следует, что единица не является точкой спектра оператора $R_{\alpha \beta}$. Отсюда и из обратимости отображений $T_{\alpha}$ и $T_{\beta}$, а также - соотношений (1П $)$ и $(4)$, следует, что

$$
T_{\alpha}^{-1}-T_{\beta}^{-1}=T_{\beta}^{-1}\left(I-R_{\alpha \beta}\right)^{-1} R_{\alpha \beta}=T_{\beta}^{-1}\left(I-R_{\beta \alpha}\right) R_{\alpha \beta}, \quad R_{\beta \alpha}=\left(A_{\beta}-A_{\alpha}\right) T_{\alpha}^{-1} .
$$

Теперь ясно, что $0 \leqslant T_{\beta}^{-1} \leqslant T_{\alpha}^{-1}$. Сравнение результатов а), б) и в) завершает доказательство.

Теорема 2. Пусть отображения $T_{\alpha}, T_{\beta}, T_{\gamma}$ класса $\Omega$ таковы, что $T_{\alpha} \leqslant T_{\beta} \leqslant T_{\gamma}$. Пусть, далее, “крайние” отображения $T_{\alpha}$ и $T_{\gamma}$ положительно обратимы. Тогда "среднее" отображсние $T_{\beta}$ также положительно обратимо и $\rho\left(R_{\alpha \gamma}\right)<1 \quad($, таким образом, мы оказываемся в условиях теоремы 1). Кроме того, $\rho\left(R_{\beta \gamma}\right)<1$ и $\rho\left(R_{\alpha \beta}\right)<1$.

ЗАмЕчАниЕ. В отличие от [6], [7], здесь все утверждения получены без предположения о компактности операторов вида $R_{\alpha \beta} \in \widetilde{Y}$.

\section{СПИСОК ЛИТЕРАТУРЫ}

[1] Иосида К. Функциональный анализ. М.: Мир, 1967. [2] Азбелев Н. В., Рахматуллина Л.Ф., Максимов В.П. Введение в теорию функционально-дифференциальных уравнений. М.: Наука, 1991. [3] Трев Ф. Введение в теорию псевдо-дифференциальных операторов и интегральных операторов Фурье. Т. 1: Псевдо-дифференциальные операторы. М.: Мир, 1984. [4] Крейн С. Г. Линейные уравнения в банаховом пространстве. М.: Наука, 1971. [5] Шефер Х. Топологические векторные пространства. М.: Мир, 1971. [6] Терентьев А.Г. // Дифференц. уравнения. 1969. Т. 5. № 9. С. 1635-1641. [7] Терентьев А. Г. // Труды Тамбовск. ин-та хим. машиностр. 1970. № 4. С. 49-60. 